\title{
Modellbildung und Temperaturregelung von Heißgasprüfständen
}

\author{
M. Freistätter, R. Bauer, N. Dourdoumas OVE, W. Rossegger
}

\begin{abstract}
Für die Entwicklung von Abgasturboladern sind Heißgasprüfstände unerlässlich. An solch einer Anlage wird die Turboladerturbine von einer Brennkammer mit einem Heißgasmassenstrom beaufschlagt. Heißgasmenge und -temperatur sind wichtige Größen während des Prüfprozesses. Zur Regelung der Heißgastemperatur wird üblicherweise ein Pl-Regler eingesetzt. Dadurch können aber nicht in allen erforderlichen Betriebspunkten befriedigende Ergebnisse erzielt werden. In dieser Arbeit wird, um eine modellbasierte Regelung zu entwerfen, ein neues thermisches Modell für die Brennkammer präsentiert. Für dieses Modell wird eine Regelstrategie vorgestellt, die den unbekannten Systemzustand benötigt. Zur Schätzung dieser Systemgröße wird ein Kalman-Filter entworfen. Messungen an der realen Anlage bescheinigen der neuen Methode ein verbessertes Führungsverhalten sowie eine bessere Störunterdrückung.
\end{abstract}

Schlüsselwörter: Abgasturbolader; Heißgasprüfstand; Temperaturregelung; Kalman-Filter

\section{Modelling and temperature control on hot gas test beds.}

Hot gas test beds are important tools for developing and testing turbochargers. In such a unit the turbine is fed by a combustion chamber. Hot gas temperature and mass flow rate are important quantities during testing. However, the use of Pl-control often leads to unsatisfactory temperature control performance. This article presents a new model-based temperature control strategy. The new model is obtained by modifying an existing thermal model of a biomass furnace. To estimate the unknown model state a Kalman-Filter is used. Measurements on the test bed prove better reference tracking as well as disturbance rejection.

Keywords: turbo charger; hot gas test bed; temperature control; Kalman-Filter

Eingegangen am 11. Jänner 2016, angenommen am 29. Juni 2016, online publiziert am 10. August 2016

(c) The Author(s) 2016. Dieser Artikel ist auf Springerlink.com mit Open Access verfügbar

\section{Einleitung}

Prüfstände sind in der Automobilindustrie zu einem wichtigen Werkzeug in der Entwicklung und Erprobung geworden. Geprüft werden nicht nur einzelne Komponenten, sondern auch Systeme mehrerer Komponenten bis hin zu vollständigen Fahrzeugen. Die grundsätzliche Anforderung für Prüfstände lautet: Wohldefinierte, reproduzierbare Bedingungen für jeden Test erzeugen. Dies stellt hohe Ansprüche an die Qualität der Prüfstandsregelung.

Zur Entwicklung von Abgasturboladern werden Heißgasprüfstände verwendet. Abbildung 1 zeigt eine schematische Darstellung solch einer Anlage. Das Funktionsprinzip ist relativ einfach: Statt der Verbrennungskraftmaschine tritt eine Brennkammer, in der das heiBe Abgas erzeugt wird, auf. Erdgas- und Luftmassenströme, $\dot{m}_{G}$ und $\dot{m}_{L}$, werden durch Versorgungsleitungen in die Brennkammer geleitet, in der die Mischung verbrannt wird. Die Menge des verbrannten Heißgases wird mittels der Stellventile in den Versorgungsleitungen von Luft, $S V_{L}$, und Erdgas, $S V_{G}$, eingestellt. Der heiße Gasmassenstrom $\dot{m}_{H G}$ wird durch ein Rohr von der Brennkammer zur Turbine T des Turboladers geleitet. Physikalische Größen, die den Zustand

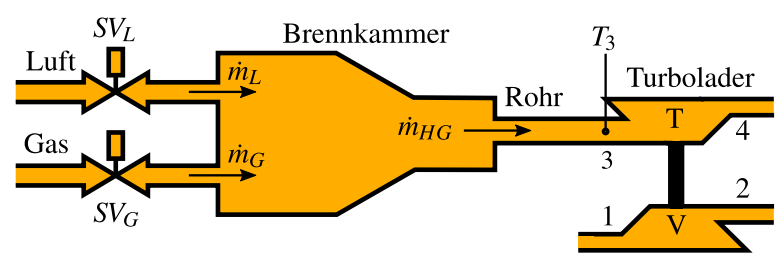

Abb. 1. Heißgasprüfstand des Heißgases am Eintritt in die Turbine beschreiben, werden mit dem Index 3 versehen. So steht $T_{3}$ für den Temperaturmesswert des Heißgases am Turbineneintritt. ${ }^{1}$ Der Heißgasmassenstrom treibt die Turbine an, welche über eine Welle mit dem Verdichter $\vee$ verbunden ist. Dieser saugt Frischluft an und bringt sie auf ein höheres Druckniveau.

Da der Verdichtungsprozess der Luft vom Expansionsprozess des Heißgases in der Turbine abhängt, haben Heißgasmassenstrom $\dot{m}_{H G}$ und -temperatur $T_{3}$ einen entscheidenden Einfluss auf das Verhalten des Turboladers. Aus diesem Grund muss ein Prüfstand (unter anderem) in der Lage sein, diese beiden physikalischen Größen schnell, exakt und voneinander unabhängig einzustellen. In Abb. 2 ist ein Blockschaltbild des Regelkreises dargestellt. Mit Strecke wird das physikalische System bestehend aus Prüfstand und Prüfling bezeich-

\footnotetext{
${ }^{1}$ Größen am Turbinenaustritt werden mit Index 4 gekennzeichnet. In analoger Weise bezeichnen die Indizes 1 und 2 den Zustand der Luft am Verdichtereinbzw. -austritt. Dies ist ein übliches Bezeichnungsschema bei der Betrachtung von Turboladern.
}

Freistätter, Markus, Institut für Regelungs- und Automatisierungstechnik, Technische Universität Graz, Inffeldgasse 21 B/l, 8010 Graz, Österreich

(E-Mail: markus.freistaetter@tugraz.at); Bauer, Robert, Kristl, Seibt \& Co GmbH, Baiernstraße 122a, 8052 Graz, Österreich (E-Mail: robert.bauer@ksengineers.at); Dourdoumas, Nicolaos, Institut für Regelungs- und Automatisierungstechnik, Technische Universität Graz, Inffeldgasse 21 B/I, 8010 Graz, Österreich (E-Mail: nicolaos.dourdoumas@tugraz.at); Rossegger, Wilfried, Kristl, Seibt \& Co GmbH, Baiernstraße 122a, 8052 Graz, Österreich

(E-Mail: wilfried.rossegger@ksengineers.at) 


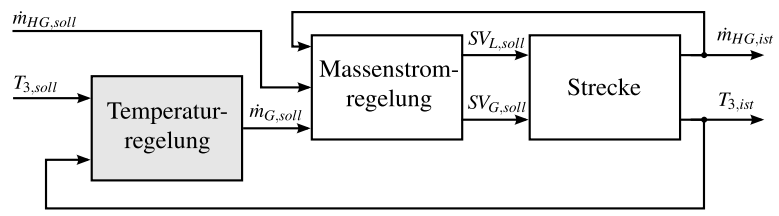

Abb. 2. Regelkreis
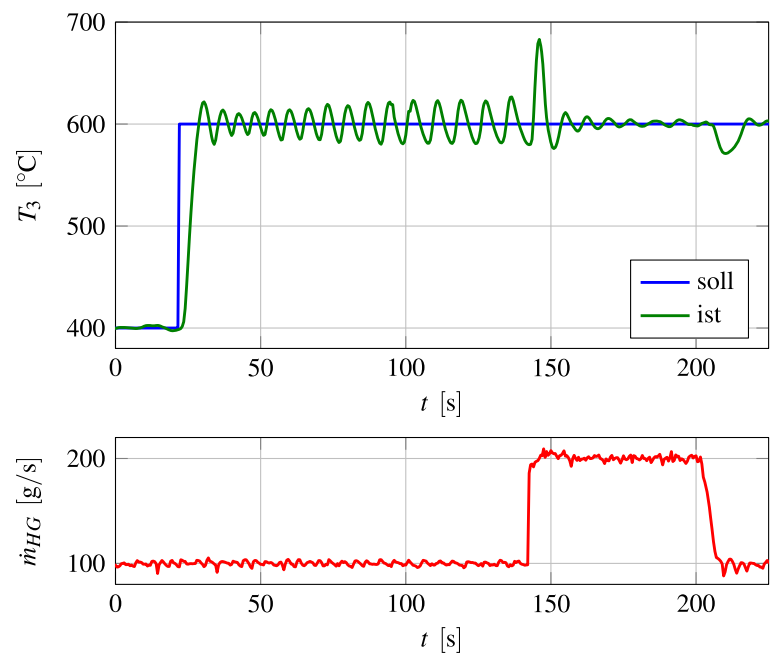

Abb. 3. Messergebnisse PI-Regler

net. Die Temperaturregelung liefert aus dem Vergleich von Solltemperatur $T_{3, \text { soll }}$ und Isttemperatur des Heißgases am Turbineneintritt $T_{3, \text { ist }}$ einen Sollwert für den Erdgasmassenstrom $\dot{m}_{G, \text { soll }}$ der unterlagerten Massenstromregelung. Zusätzlich erhält diese einen Sollwert für den gesamten Heißgasmassenstrom, $\dot{m}_{H G, s o l l}$. Der Sollwert kann manuell vorgegeben werden, es ist aber auch eine Vorgabe durch einen überlagerten Drehzahlregler möglich. Aus dem Heißgasmassenstromsollwert wird im Regler unter Berücksichtigung des Erdgasmassenstromsollwerts ein Sollwert für den Luftmassenstrom bestimmt. Der Messwert des Heißgasmassenstroms, $\dot{m}_{H G, i s t}$, wird dem Regler rückgeführt. Die Stellgrößen der Massenstromregelung sind die Sollpositionen der Stellventile der Luft- $\left(S V_{L, \text { soll }}\right)$ und Gasversorgungen $\left(S V_{G, \text { soll }}\right)$. Auf die Massenstromregelung selbst wird in weiterer Folge nicht eingegangen. Ihr Verhalten wird als ideal angenommen.

In vorliegender Arbeit wird eine neue Methode zur Temperaturregelung von Heißgasprüfständen vorgestellt. Versuche am Prüfstand mit einem PI-Regler führten zu einem unbefriedigenden Ergebnis, siehe Abb. 3. Darin ist zum einen die Antwort des geregelten Systems auf eine Änderung des Temperatursollwerts am Turbineneintritt ersichtlich. Bei konstantem Heißgasmassenstrom $\dot{m}_{H G}$ durch Brennkammer und Turbine wird der Temperatursollwert von $400^{\circ} \mathrm{C}$ auf $600^{\circ} \mathrm{C}$ erhöht. Hierbei kommt es zu einem oszillierendem Temperaturverlauf, dessen Amplitude nicht kleiner wird. Im Prüfbetrieb ist solch ein Verhalten nicht akzeptabel!

Zum anderen wird etwa 120 s nach einer Erhöhung des Temperatursollwerts der Heißgasmassenstrom von $100 \mathrm{~g} / \mathrm{s}$ auf $200 \mathrm{~g} / \mathrm{s}$ geändert. Dabei kommt es zu einer Abweichung der gemessenen Temperatur vom Sollwert von etwa $100^{\circ} \mathrm{C}$. Auch bei der anschließenden Reduktion des Massenstromes kommt es zu einer deutlichen Temperaturabweichung. Diese deutlichen Temperaturschwankungen sind ebenfalls unerwünscht.

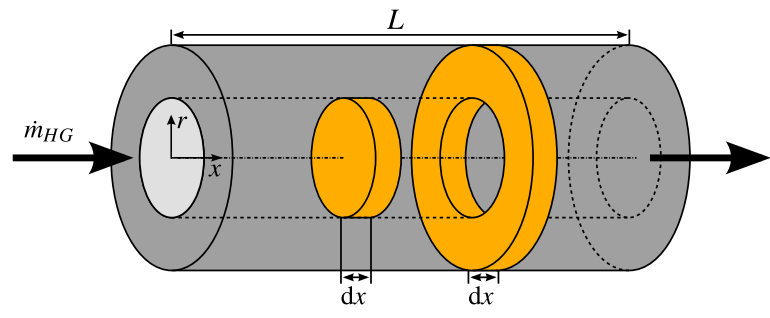

Abb. 4. Strömungsprozess in Rohr mit Isolierung

Zur Verbesserung des Führungsverhaltens und der Störunterdrückung wird in vorliegender Arbeit ein modellbasierter Ansatz vorgeschlagen. Im entwickelten Modell wird die Abhängigkeit des Temperaturverhaltens vom Massenstrom berücksichtigt. Mit dem neuen Regelkonzept kann auch die Überschwingweite der Temperatur bei sprungförmigen Änderungen des Sollwerts reduziert werden.

Die Methode basiert auf Erkenntnissen in [2] zur Modellbildung und Regelung einer Biomassefeuerungsanlage. Beim vorgeschlagenen Konzept wird eine "gestörte" Systemgröße benötigt. Aus diesem Grund wird zu deren Schätzung ein Kalman-Filter eingesetzt. Vergleiche mit dem ursprünglichen Regelkonzept zeigen, dass das neue bezüglich Führungsverhaltens und Einfluss von Massenstromänderungen überlegen ist.

\section{Modellbildung}

In diesem Abschnitt wird eine Zusammenfassung der Erkenntnisse in [5] und [2] präsentiert. Das Ergebnis der Modellbildung ist ein einfaches, allgemein gültiges Modell zur Beschreibung des thermischen Verhaltens von Brennkammer und Rohr.

\subsection{Genaue Betrachtung}

Die Brennkammer und das Verbindungsrohr zum Turbolader werden in erster Näherung als ein Rohrstück mit Isolierung betrachtet. Die Isolierung sorgt für eine Reduktion der Energieabgabe an die Umgebung. Der Strömungsprozess in solch einem zylindrischen Rohr ist in Abb. 4 dargestellt. Die Geometrie des Rohres ist durch dessen Länge $L$ und Durchmesser, sowie die Dicke der Isolationsschicht gegeben. Der Heißgasmassenstrom $\dot{m}_{H G}$ tritt an Position $x=0$ in das Rohr ein und bei $x=L$ aus. Die physikalischen Größen, die den Zustand des Heißgases beschreiben, weisen im Allgemeinen eine Abhängigkeit von der Position $x$, der Zeit $t$ und eine radiale Abhängigkeit $r$ auf.

Letztere kann unter der Annahme turbulenter Strömung vernachlässigt werden, da eine turbulente Strömung für eine homogene Durchmischung des Fluids in jedem Rohrquerschnitt sorgt $[5,6]$. Die Größen sind nur noch von der Zeit $t$ und der Längsrichtung $x$ abhängig. Der Übersichtlichkeit halber werden diese Abhängigkeiten nicht angeführt. Die Gleichungen zur Beschreibung der in Abb. 4 hervorgehobenen Volumenelemnte lauten:

$$
\begin{aligned}
& \frac{\partial u_{H G}}{\partial t}+\frac{\partial s_{h, H G}}{\partial x}+s_{q, H G}=\frac{\partial}{\partial x}\left(\lambda_{H G} \frac{\partial T_{H G}}{\partial x}\right) \\
& \frac{\partial u_{l}}{\partial t}+\frac{\partial s_{h, l}}{\partial x}+s_{q, l}=\frac{\partial}{\partial x}\left(\lambda_{l} \frac{\partial T_{I}}{\partial x}\right)
\end{aligned}
$$

Die Indizes $H G$ und $/$ in obigen Gleichungen bezeichnen die jeweiligen Größen für Heißgas und Isolationsschicht. Die erscheinenden Größen haben folgende Bedeutung: Die Energiedichte der inneren Energie wird durch $u$ gekennzeichnet, $s_{h}$ steht für die Enthalpiestromdichte, $s_{q}$ bezeichnet die Wärmestromdichte des mit der Umgebung ausgetauschten Wärmestroms, $\lambda$ die Wärmeleitfähigkeit des Stoffes und $T$ die thermodynamische Temperatur. Der Ausdruck 
$\frac{\partial u}{\partial t}$ beschreibt die Änderung der inneren Energie innerhalb des Volumenelements über der Zeit. Im zweiten Term, $\frac{\partial S_{h}}{\partial x}$, wird die Änderung der Energie aufgrund der Bewegung des Stoffes berücksichtigt. Vervollständigt wird die linke Seite durch $s_{q}$, den Energieaustausch mit der Umgebung. Auf der rechten Seite der Gleichungen findet im Ausdruck $\frac{\partial}{\partial x}\left(\lambda \frac{\partial T}{\partial x}\right)$ die Wärmeleitung in axialer Richtung Berücksichtigung.

Aufgrund von Vereinfachungen, wie etwa Verwendung des Stoffmodells des idealen Gases oder Vernachlässigung axialer Wärmeleitung ergibt sich folgendes Modell:

$$
\begin{aligned}
& \frac{\partial T_{H G}}{\partial x}+\kappa_{1}\left(T_{H G}-T_{l}\right)=0 \\
& \frac{\partial T_{l}}{\partial t}+\kappa_{2}\left(T_{l}-T_{H G}\right)=0 .
\end{aligned}
$$

Die Funktionen $\kappa_{1}$ und $\kappa_{2}$ sind unter anderem vom Massenstrom oder der Heißgastemperatur abhängig. Dieses Modell stellt bereits eine Vereinfachung dar, ist jedoch für einen modellbasierten Reglerentwurf schlecht geeignet. Es ist eine weitere Vereinfachung dieses Modells erstrebenswert!

Im Grunde ist nur das Übertragungsverhalten von der Heißgastemperatur am Rohreintritt zur Heißgastemperatur am Rohraustritt von Interesse. Die Temperatur am Rohreintritt, $T_{H G}(x=0, t)$, wird als adiabate Verbrennungstemperatur $T_{A V}$ [1] bezeichnet, die Temperatur am Rohraustritt, $T_{H G}(x=L, t)$, ist die Temperatur am Turbineneintritt $T_{3}$.

\subsection{Adiabate Verbrennungstemperatur}

Ausgehend von der Leistungsbilanzgleichung für eine adiabate Brennkammer

$$
\dot{m}_{G} h_{G}\left(T_{G}\right)+\dot{m}_{L} h_{L}\left(T_{L}\right)=\dot{m}_{H G} h_{H G}\left(T_{A V}\right)
$$

kann ein einfacher Zusammenhang zwischen der adiabaten Verbrennungstemperatur und dem Erdgasmassenstrom hergestellt werden. In Gl. (4) sind die Enthalpien $h$ von Gas, Luft und Heißgas durch die entsprechenden Indizes gekennzeichnet. Sie sind abhängig von den jeweiligen Temperaturen $T$ der Stoffe.

Es wird näherungsweise angenommen, dass die Enthalpie des Erdgases durch dessen Heizwert $H_{i}$ bestimmt wird,

$$
h_{\mathrm{G}} \approx H_{i} \text {. }
$$

Zusätzlich wird die vergleichsweise geringe Enthalpie der Verbrennungsluft vernachlässigt $\left(h_{L}=0\right)$. Mit der Massenbilanz

$$
\dot{m}_{H G}=\dot{m}_{G}+\dot{m}_{L}
$$

erhält man nach einigen Umformungen unter Verwendung der mittleren spezifischen Wärmekapazität des Heißgases, $C_{p, H G}$,

$$
\begin{aligned}
& h_{H G}=c_{p, H G}\left(T_{A V}-T_{0}\right) \\
& \dot{m}_{G}=\frac{\dot{m}_{L} h_{H G}}{H_{i}-h_{H G}} .
\end{aligned}
$$

Damit ist ein Zusammenhang zwischen der adiabaten Verbrennungstemperatur $T_{A V}$ und dem Gasmassenstrom $\dot{m}_{G}$, der Stellgröße des Temperaturreglers gegeben.

Die gewünschte adiabate Verbrennungstemperatur wird vom Massenstromregler in Form eines Gasmassenstroms und eines passenden Luftmassenstroms eingestellt. Die Verbrennung geschieht überstöchiometrisch und mit variablem Verbrennungsluftverhältnis.

Durch Umformung der Gl. (6) und (7) ist es umgekehrt auch möglich, aus Massenstrommesswerten die adiabate Verbrennungstemperatur zu berechnen. Sie ändert sich folglich in Abhängigkeit der Luft- und Gasmassenströme, wie etwa auch in [1] beschrieben.

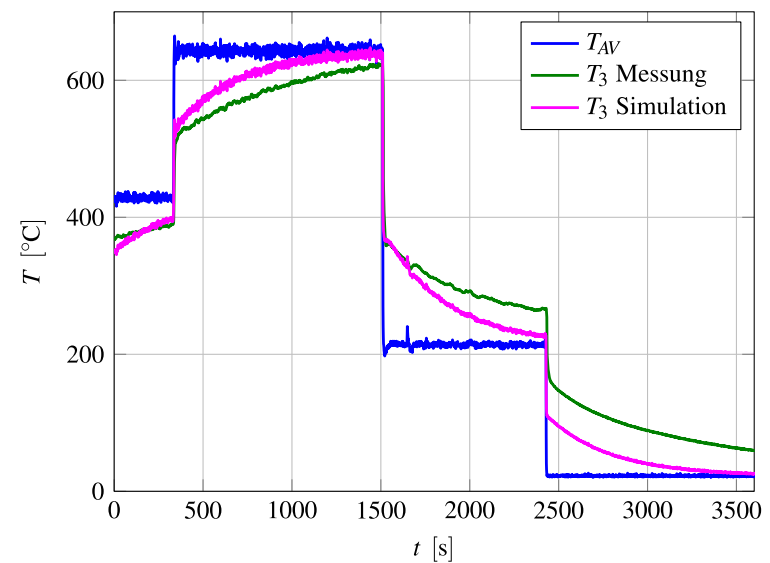

Abb. 5. Simulationsergebnisse für Modell (8)

\subsection{Ein einfaches Modell}

Wie zuvor erwähnt ist die Temperatur am Austritt des Rohrs die Heißgastemperatur am Eintritt in die Turbine. Sie ist die Messgröße für die Temperaturregelung. Da nur der Zusammenhang zwischen den Temperaturen am Rohrein- und -austritt gesucht ist, ist eine einfachere Beschreibung möglich. Es ist bemerkenswert, dass dieser Zusammenhang durch ein Modell der Art

$$
\frac{\mathrm{d} x}{\mathrm{~d} t}=-a x+a u, \quad y=c x+(1-c) u
$$

mit den Parametern a und $c$ beinahe exakt beschrieben werden kann, wie in [2] gezeigt wird. Es handelt sich dabei nicht um ein semi-empirisches Modell, sondern es ist allgemein gültig!

Die adiabate Verbrennungstemperatur $T_{A V}$ stellt die EingangsgröBe $u$ dar, die Ausgangsgröße $y$ ist durch die Temperatur des Heißgases am Ende des Rohrs $T_{3}$ gegeben. Die sogenannte Zustandsgröße $x$ kann als mittlere Isolationstemperatur interpretiert werden. Sie ist nur mehr eine Funktion der Zeit und zeigt keine örtliche Abhängigkeit!

Das Übertragungsverhalten weist eine starke Abhängigkeit vom Heißgasmassenstrom auf. Aus diesem Grund sind die Parameter a und c Funktionen des Massenstroms:

$$
a\left(\dot{m}_{H G}\right)=c_{a} \dot{m}_{H G}^{0.8}, \quad c\left(\dot{m}_{H G}\right)=c_{c} \dot{m}_{H G}^{-0.15}
$$

Die Exponenten 0.8 und -0.15 in obigen Gleichungen entstammen der nahezu exakten Herleitung. Die Koeffizienten $c_{a}$ und $c_{c}$ werden aus Messdaten ermittelt.

\section{Modellverifikation}

Zur Überprüfung der Eignung des Modells (8) wurden aus Messdaten von Versuchen die Koeffizienten $c_{a}$ und $c_{c}$ bestimmt und eine Simulationsstudie durchgeführt. In Abb. 5 sind gemessene und simulierte Werte gegenübergestellt. Es wird bei konstantem Brennkammermassenstrom das Systemverhalten bei einer sprunghaften Änderung der Eingangsgröße $T_{A V}$ mit dem realen thermischen Verhalten von Brennkammer und Rohr verglichen. Das asymptotische Verhalten wird adäquat nachgebildet. Genauere Betrachtungen offenbaren jedoch einige Mängel. In einer vergrößerten Ansicht in Abb. 6 ist ersichtlich, dass sich gemessener und simulierter Temperaturverlauf in den ersten 20 Sekunden nach dem Sprung der adiabaten Verbrennungstemperatur deutlich unterscheiden. Die gemessene Temperatur reagiert auf den Sprung deutlich langsamer.

Bezüglich des Einflusses des Massenstroms auf das thermische Verhalten fallen zwei zusätzliche Unzulänglichkeiten auf. Wie Abb. 7 


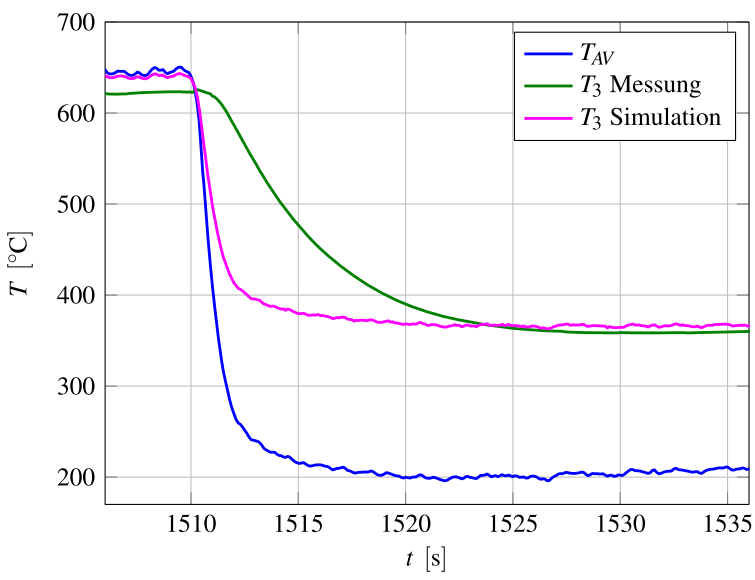

Abb. 6. Simulationsergebnisse für Modell (8) (vergrößerte Ansicht)
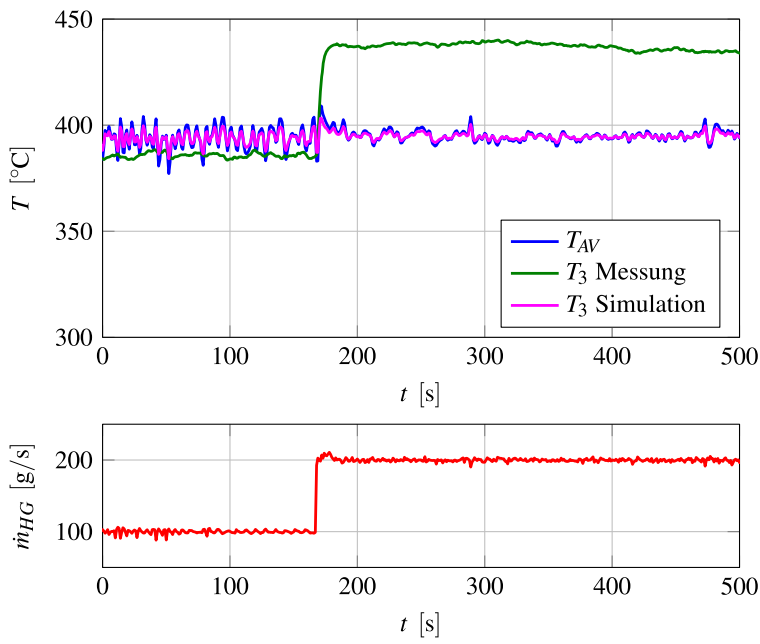

Abb. 7. Simulationsergebnisse bei Massenstromsprung

zeigt, ändert sich die Temperatur am Sensor im eingeschwungenen Zustand bei einem Massenstromsprung. Die simulierte Temperatur zeigt auf diese Änderung keine Auswirkung. Ein weiteres Problem ist Ansatz (9) für den Parameter c. Durch die aus Messdaten errechneten Werte erkennt man (siehe Abb. 8), dass der Ansatz für vorliegende Anwendung ungeeignet ist.

Bei einer sprunghaften Änderung des Massenstroms wie in Abb. 7 verläuft die eingeschwungene Temperatur am Turbineneintritt nach dem Massenstromsprung oberhalb der adiabaten Verbrennungstemperatur. Ein kurzzeitiger Temperaturanstieg aufgrund einer Massenstromänderung kann durch die Konstruktion des Brenners erklärt werden, (siehe Abb. 9). Um die Brennkammer zu kühlen wird ein Teil der Verbrennungsluft mehrmals am Brennermantel entlang geführt. Dieser Teil tritt vorgewärmt in die Brennkammer ein. Bei einer Massenstromänderung ändert sich der Anteil dieser vorgewärmten Luft am stärksten, wodurch es zu einer Temperaturänderung kommt.

Die Brennerisolation ist auch der Grund, weshalb Strahlungsvorgänge nicht explizit berücksichtigt werden. Zwischen der heißen Brennkammer und deren Isolation findet zwar ein Strahlungsaustausch statt, wodurch die Isolationsschicht erwärmt wird. Da die Isolation aber von einem Teil der Verbrennungsluft durchströmt wird, wird diese Energie zu einem großen Anteil wieder in die Brennkammer rückgeführt. Die Isolationsschicht ist deutlich kühler als die Brennkammer, der Temperaturunterschied zur Umgebung nicht

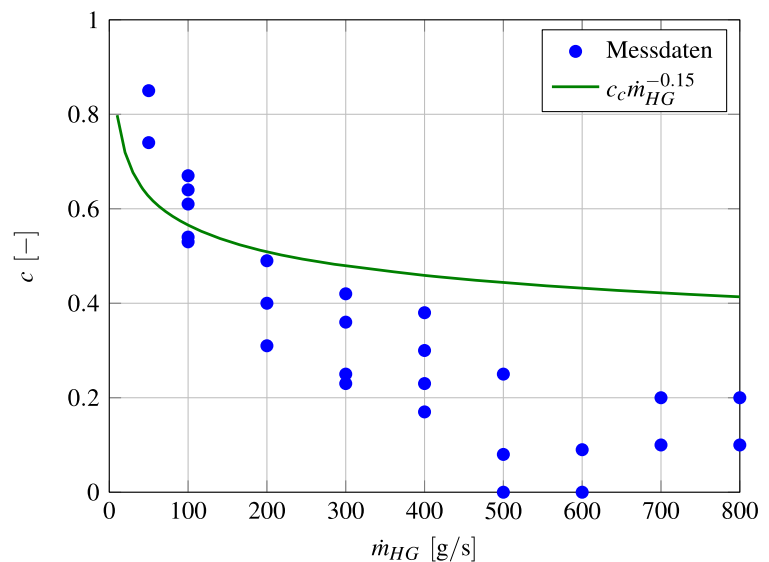

Abb. 8. Parameter c nach Ansatz (9)

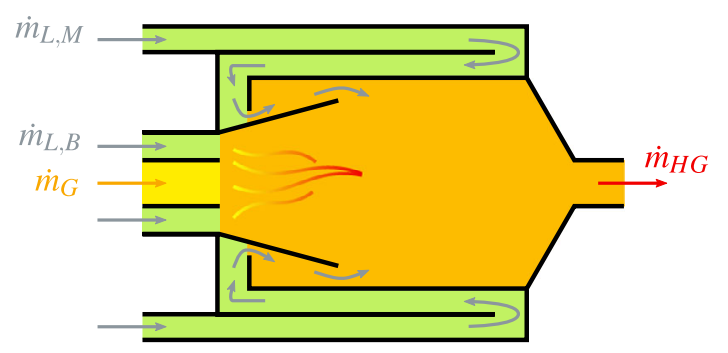

Abb. 9. Schema der Brennkammer

mehr so groß. Aus diesem Grund wird der Strahlungsaustausch zwischen Isolation und Umgebung vernachlässigt.

Nach physikalischen Gesichtspunkten ist jedoch nicht nachvollziehbar, dass die Heißgastemperatur stationär über der adiabaten Verbrennungstemperatur liegt. Dies bedeutet, dass die Berechnung der adiabaten Verbrennungstemperatur nach GI. (7) aufgrund etlicher Vereinfachungen ungenau ist.

Eine exakte Bestimmung der adiabaten Verbrennungstemperatur ist aufgrund der schwankenden Zusammensetzung des Erdgases nicht möglich. Aus diesem Grund wird auf eine aufwändige Analyse verzichtet. Stattdessen wird der einfache Zusammenhang (7) weiterverwendet. Es zeigt sich, dass der beschriebene Effekt keinen nennenswerten Einfluss auf die Qualität der Regelung hat.

Aus der Verifikation des Modells (8) ergeben sich somit drei Bereiche, in denen eine Verbesserung nötig ist:

1. Einfluss eines Massenstromsprunges auf die Temperatur.

2. Temperaturverhalten kurz nach einem Sprung der adiabaten Verbrennungstemperatur.

3. Mathematischer Ansatz für Parameter c.

\section{Modellerweiterung}

Die drei erwähnten Mängel werden nun durch Adaptierung des ursprünglichen Modells beseitigt, um die Qualität des Modells für den Reglerentwurf zu verbessern.

\section{$4.1 \dot{m}$-Sprung-Modell}

Der größte Unterschied zwischen realem und modelliertem Temperaturverhalten zeigt sich bei Massenstromänderungen im eingeschwungenen Zustand (siehe Abb. 7). Bei stationärem Verhalten, das heißt $\frac{d x}{d t}=0$, ergibt sich für das System nach Gl. (8),

$$
\frac{\mathrm{d} x}{\mathrm{~d} t}=-a x+a u \quad y=c x+(1-c) u,
$$


der Systemzustand

$$
x_{\infty}=T_{l, m, \infty}=\frac{a}{a} u_{0}=T_{A V, 0} .
$$

Für die Ausgangsgröße gilt

$$
y_{\infty}=T_{3, \infty}=c x_{\infty}+(1-c) u_{0}=T_{A V, 0} .
$$

Offensichtlich hat der Massenstrom keinen Einfluss auf die Temperatur am Sensor im eingeschwungenen Zustand.

Ein naheliegender Wunsch ist es, die Komplexität des Modells möglichst niedrig zu halten. Aus diesem Grund ist man bestrebt, das thermische Verhalten bei einer Änderung des Massenstroms durch ein Modell erster Ordnung nachzubilden. Um das Verhalten an die Realität anzupassen, verändert man die Modellparameter. Aus Abb. 7 ist ersichtlich, dass eine Vergrößerung des Massenstromes zu einer erhöhten Temperatur am Turbineneintritt führt. Sinkt der Massenstrom, so sinkt die Temperatur ebenfalls. Im eingeschwungenen Zustand nach dem Massenstromsprung muss die Temperatur am Turbineneintritt jedoch, da Verluste nicht berücksichtigt werden, wieder der adiabaten Verbrennungstemperatur entsprechen,

$$
T_{3, \infty} \stackrel{!}{=} T_{A V, 0}
$$

Diese Forderung ist darin zu begründen, dass bei einer Massenstromänderung hauptsächlich der Anteil der Luft verändert wird, mit dem der Brennkammermantel auch gekühlt wird. Wird dieser Anteil erhöht, so wird kurzzeitig mehr Luft mit einer höheren Temperatur in die Brennkammer geleitet. Dies führt zu einem Anstieg der Temperatur. Im eingeschwungenen Zustand muss sich die Temperatur aber wieder auf den stationären Wert einpendeln. Das veränderte Verhalten muss daher im Zustand des Systems berücksichtigt werden.

Die ursprüngliche Zustandsgleichung des Systems (8) wird zu

$$
\frac{\mathrm{d} x}{\mathrm{~d} t}=-a x+b u
$$

mit dem neuen Parameter $b$ verändert. Letzterer ist vom Massenstrom abhängig. Der eingeschwungene Zustand ändert sich zu

$$
x_{\infty}=\frac{b}{a} u_{0} .
$$

Damit Anforderung (12) weiterhin erfüllt ist, wird die Ausgangsgleichung des Systems (8) ebenfalls modifiziert:

$$
y=\frac{a}{b} c x+(1-c) u
$$

Die Multiplikation des ersten Terms mit dem Faktor $\frac{a}{b}$ bewirkt, dass $T_{3}$ im eingeschwungenen Zustand wieder $T_{A V}$ entspricht. Gleichungen (13) und (15) bilden das sogenannte $\dot{m}$-Sprung-Modell.

Für den Parameter $b$ wird mit

$$
b\left(\dot{m}_{H G}\right)=c_{a} \dot{m}_{H G}^{q_{b}}
$$

ein ähnlicher Ansatz wie für Parameter a verwendet.

Ein Vergleich des neuen Modells mit dem ursprünglichen in Abb. 10 zeigt die Verbesserung des Verhaltens bei einem Massenstromsprung: Es ändert sich nun auch die Temperatur am Sensor sprunghaft, um sich im Anschluss wieder asymptotisch dem Wert im eingeschwungenen Zustand anzunähern. Das Modell ist damit zwar nicht dazu in der Lage, das stationäre Verhalten exakt zu beschreiben, dies ist für den Reglerentwurf aber auch nicht unbedingt nötig! Viel wichtiger ist die Fähigkeit, die Auswirkungen von schnellen Massenstromänderungen auf das dynamische Verhalten der Heißgastemperatur gut abzubilden. Für den späteren Reglerentwurf ist diese Modelleigenschaft von besonderer Bedeutung.
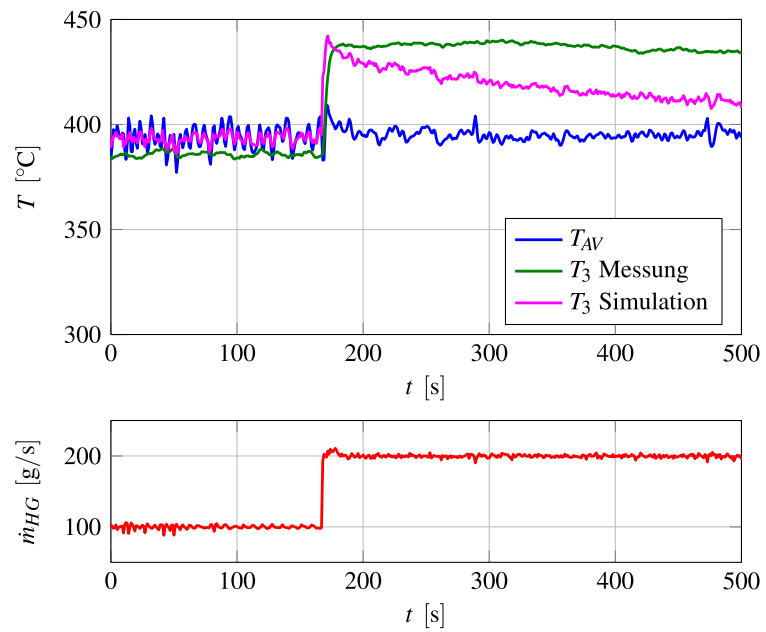

Abb. 10. Simulationsergebnisse für das $\dot{\boldsymbol{m}}$-Sprung-Modell

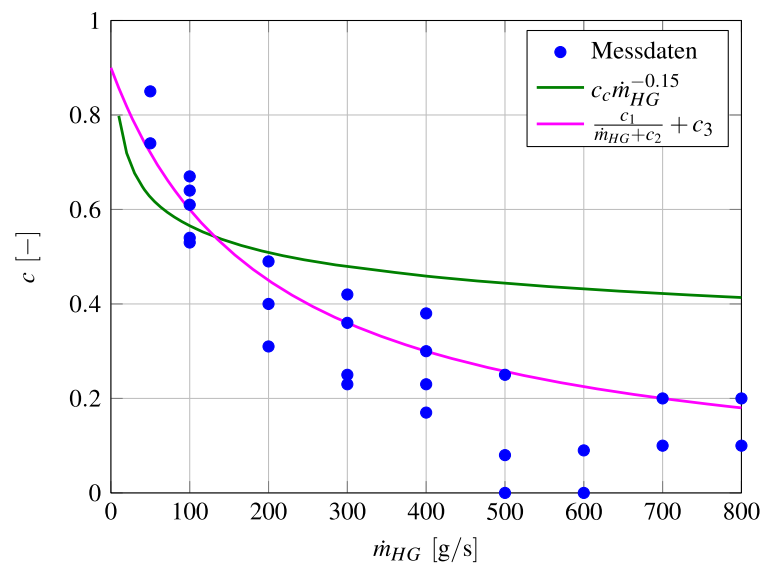

Abb. 11. Neuer Ansatz für Parameter c

\subsection{Modellparameter}

Wie bereits in Abschn. 3 erwähnt, ist der in [2] vorgeschlagene Ansatz des Parameters $\mathrm{c}$ im vorliegenden Fall ungeeignet. Es wird ein neuer Ansatz für $c=c\left(\dot{m}_{H G}\right)$ gesucht. Die gesuchte Funktion soll monoton mit steigendem Massenstrom fallen und für große Massenströme gegen einen konstanten Wert streben. Zusätzlich soll sie bei einem Massenstrom vom Wert Null konstant sein.

Die rationale Funktion

$$
c\left(\dot{m}_{H G}\right)=\frac{c_{1}}{\dot{m}_{H G}+c_{2}}+c_{3}
$$

mit den Konstanten $c_{1}, c_{2}$ und $c_{3}$ erfüllt diese Anforderungen. Abbildung 11 zeigt einen Vergleich mit dem ursprünglichen Ansatz nach (9). Die Übereinstimmung mit den Messdaten wird durch den neuen Ansatz signifikant verbessert.

\subsection{Berücksichtigung des dynamischen Verhaltens des \\ Temperatursensors}

Der letzte offene Punkt bei der Verbesserung des Temperaturmodells betrifft die mit Abb. 6 aufgeworfene Temperaturänderung bei sprunghafter Änderung der Eingangsgröße. Die Temperatur des Modells reagiert im Vergleich zur Realität deutlich schneller auf solch eine Änderung. Die Ursache liegt im Verhalten des Temperatursensors. Wegen dessen thermischer Trägheit kommt es zu der in Abb. 6 dargestellten Messabweichung. 


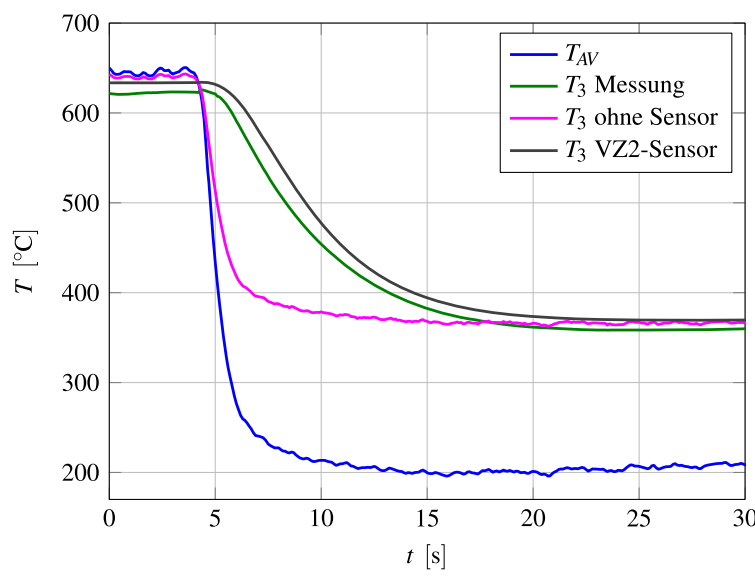

Abb. 12. Simulationsergebnisse des Modells bei $T_{A V}$-Sprung

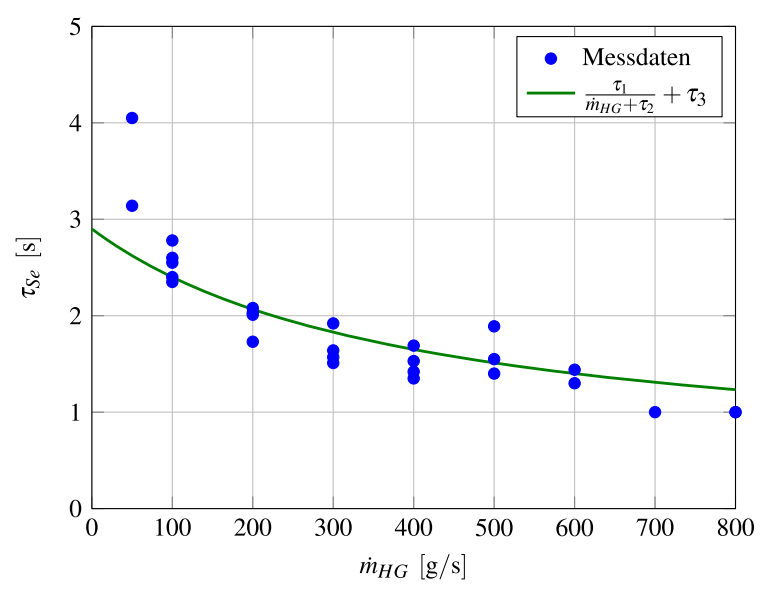

Abb. 13. Abhängigkeit der Sensorzeitkonstante vom Massenstrom

Bei dem verwendeten Temperatursensor handelt es sich um ein Thermoelement. Ein Thermoelement steht nicht direkt in Kontakt zum vorbeiströmenden Heißgas, sondern ist durch ein Schutzrohr isoliert. Es besitzt somit zwei thermische Trägheiten, weshalb das Differentialgleichungssystem zweiter Ordnung

$$
\begin{aligned}
\frac{\mathrm{d} T_{S c h}}{\mathrm{~d} t} & =\frac{1}{\tau_{S e}}\left(T_{H G}-T_{S c h}\right) \\
\frac{\mathrm{d} T_{S e}}{\mathrm{~d} t} & =\frac{1}{\tau_{S e}}\left(T_{S c h}-T_{S e}\right) .
\end{aligned}
$$

zur Beschreibung des dynamischen Verhaltens vorgeschlagen wird [7]. $T_{\text {Sch }}$ symbolisiert die Temperatur des Schutzrohrs, $T_{S e}$ die des Sensors. Der Einfachheit halber wird die Zeitkonstante $\tau_{S e}$ für beide Gleichungen identisch angenommen. Der Verlauf der Messgröße wird, wie in Abb. 12 als VZ2-Sensor ${ }^{2}$ ersichtlich, zufriedenstellend abgebildet.

Um der in Abb. 13 dargestellten Abhängigkeit der Sensorzeitkonstanten vom Massenstrom Rechnung zu tragen, kann derselbe Ansatz wie bereits für den Modellparameter c nach Gl. (17) verwendet werden. Mit den Parametern $\tau_{1}, \tau_{2}$ und $\tau_{3}$ wird für die Sensorzeitkonstante

$$
\tau_{S e}=\frac{\tau_{1}}{\dot{m}_{H G}+\tau_{2}}+\tau_{3}
$$

${ }^{2}$ Verzögerungsglied 2. Ordnung
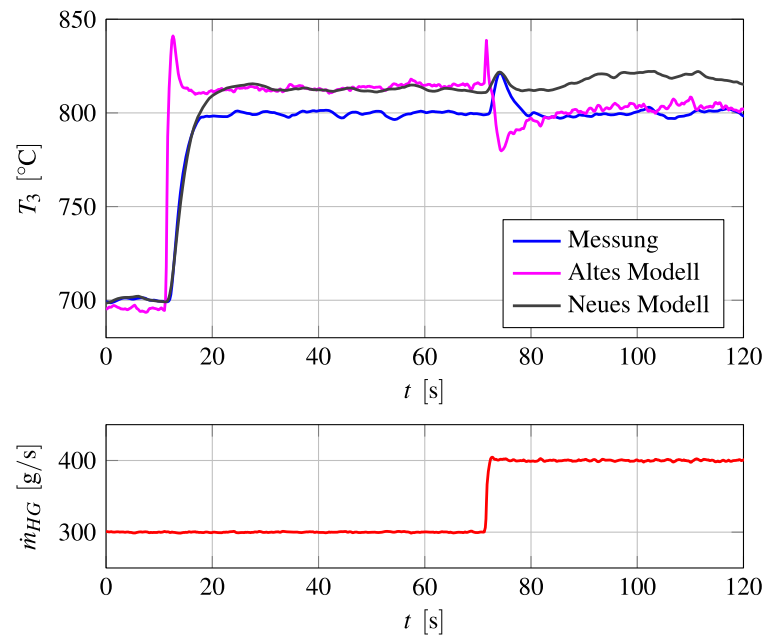

Abb. 14. Vergleich der Modelle

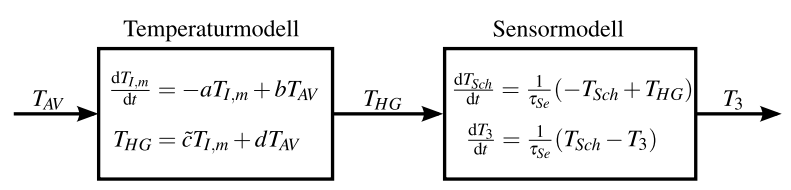

Abb. 15. Blockschaltbild Gesamtsystem

angesetzt. Die gute Übereinstimmung mit den aus den Messdaten errechneten Werten ist in Abb. 13 ersichtlich.

In der Gegenüberstellung des ursprünglichen Modells nach [2] und der erweiterten Version in Abb. 14 sind die erzielten Verbesserungen ersichtlich. Alle in Abschn. 3 aufgeworfenen Problemstellen konnten beseitigt werden. Die wesentlichen thermischen Vorgänge und Abhängigkeiten werden nun erfasst. Damit ist das Modell für den Reglerentwurf gut geeignet.

\section{Regelung}

Zuerst wird das mathematische Modell der Gl. (13) und (15) betrachtet:

$$
\frac{\mathrm{d} T_{l, m}}{\mathrm{~d} t}=-a T_{l, m}+b T_{A V} \quad T_{H G}=\tilde{c} T_{l, m}+\mathrm{d} T_{A V}
$$

Als Ausgangsgröße wird nun die tatsächliche Heißgastemperatur $T_{H G}$ interpretiert. Für die Parameter werden dabei die Abkürzungen

$$
\begin{aligned}
& \tilde{c}=\frac{a}{b} c, \\
& d=1-c
\end{aligned}
$$

eingeführt. Durch Reihenschaltung der Systeme Temperaturmodell nach (21) und Temperatursensor nach (18) und (19) (siehe Abb. 15) ergibt sich ein Gesamtsystem der Form

$$
\begin{aligned}
& \frac{\mathrm{d} \mathbf{x}}{\mathrm{d} t}=\mathbf{A} \mathbf{x}+\mathbf{b} u \\
& y=\mathbf{c}^{T} \mathbf{x} .
\end{aligned}
$$

Die adiabate Verbrennungstemperatur ist die Eingangsgröße des Gesamtsystems, $u=T_{A V}$. Die Ausgangsgröße des Temperaturmodells ist die Eingangsgröße des Sensormodells. Dessen Ausgangsgröße ist der Temperaturmesswert am Turbineneintritt, $y=T_{S e}=T_{3}$. 
Dadurch ergeben sich die Systemparameter

$$
\mathbf{A}=\left[\begin{array}{ccc}
-a & 0 & 0 \\
\frac{\tilde{c}}{\tau_{S e}} & -\frac{1}{\tau_{S e}} & 0 \\
0 & \frac{1}{\tau_{S e}} & -\frac{1}{\tau_{S e}}
\end{array}\right], \quad \mathbf{b}=\left[\begin{array}{c}
b \\
\frac{d}{\tau_{S e}} \\
0
\end{array}\right], \quad \mathbf{c}^{T}=\left[\begin{array}{lll}
0 & 0 & 1
\end{array}\right] .
$$

Im Zustandsvektor

$$
\mathbf{x}=\left[T_{l, m} T_{S c h} T_{3}\right]^{T}
$$

sind die Temperaturen des $\dot{m}$-Sprung-Modells, des Schutzrohrs und des Temperatursensors enthalten.

Für die Regelung des gesamten Heißgasprüfstands wird ein Digitalrechner eingesetzt, der die Regelungsalgorithmen von Massenstrom- und Temperaturregelung in Echtzeit abarbeitet. Aus diesem Grund werden die Regelungsalgorithmen in zeitdiskreter Form entworfen.

Unter Zugrundelegung der Diskretisierungszeit (Abtastzeit) $T_{d}$ wird zunächst das zeitkontinuierliche Modell nach Gl. (22) durch das zeitdiskrete System

$$
\begin{aligned}
& \mathbf{x}_{i+1}=\boldsymbol{\Phi} \mathbf{x}_{i}+\mathbf{h} u_{i} \\
& y_{i}=\mathbf{c}^{T} \mathbf{x}_{i}
\end{aligned}
$$

ersetzt. Die Kennzeichnung der vorkommenden Größen mit dem Index $i$ ist eine vereinfachende Schreibweise für die an den jeweiligen Abtastzeitpunkten ausgewerteten Zeitfunktionen $f(t)$ :

$$
f_{i}:=f\left(i T_{d}\right), \quad i=0,1,2, \ldots
$$

Die Systemparameter der Zustandsgleichung (24) werden bei stückweise konstanter Eingangsfunktion durch

$$
\boldsymbol{\Phi}=e^{\mathbf{A} T_{d}}, \quad \mathbf{h}=\int_{0}^{T_{d}} e^{\mathbf{A} \tau} \mathbf{b} d \tau
$$

bestimmt.

Das reale Verhalten des Systems weicht immer vom modellierten ab. Zur Beschreibung von in der Realität auftretenden Störungen und Ungenauigkeiten wird das Modell mithilfe der Größen w und $v$ erweitert:

$$
\begin{aligned}
& \mathbf{x}_{i+1}=\boldsymbol{\Phi} \mathbf{x}_{i}+\mathbf{h} u_{i}+\mathbf{w}_{i} \\
& y_{i}=\mathbf{c}^{T} \mathbf{x}_{i}+v_{i}
\end{aligned}
$$

Diese Größen sind stochastischer Natur. Es wird angenommen, dass sie stationäres weißes Rauschen und unkorreliert sind. Zusammengefasst lauten die Eigenschaften:

$$
\begin{array}{ll}
E\left\{\mathbf{w}_{i}\right\}=\mathbf{0}, & E\left\{\mathbf{w}_{i} \mathbf{w}_{j}^{T}\right\}=\mathbf{Q} \delta_{i j} \\
E\left\{v_{i}\right\}=0, & E\left\{v_{i} v_{j}\right\}=R \delta_{i j} \\
E\left\{\mathbf{w}_{i} v_{j}\right\}=\mathbf{0} &
\end{array}
$$

Mit $\delta_{i j}$ wird das Kronecker-Symbol bezeichnet. Ferner weisen das sogenannte Zustandsrauschen $\mathbf{w}$ und Messrauschen $v$ mit dem Anfangswert $\mathbf{x}_{0}$ keine Korrelation auf.

\subsection{Regelstrategie}

Die Strategie zur Temperaturregelung verfolgt den Ansatz, das erarbeitete Modellwissen einzusetzen. In der Ausgangsgleichung des Systems (21) ist ein direkter Zusammenhang zwischen Eingangsund -ausgangsgröße gegeben. Ist der Wert der ersten Komponente des Systemzustands, $x_{1, i}$, bekannt, so kann für eine gewünschte Temperatur $T_{3, i}=y_{i}$ die gesuchte Stellgröße $T_{A F, i}=u_{i}$ sofort bestimmt werden! Der erste Systemzustand entspricht der mittleren

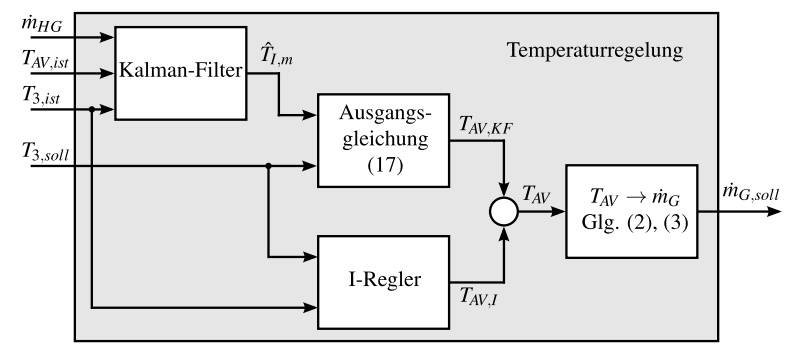

Abb. 16. Blockschaltbild Regler, vgl. Abb. 2

Isolationstemperatur. Diese Temperatur kann allerdings nicht messtechnisch ermittelt werden, da es sich um eine "modellhafte" mittlere Temperatur handelt. Aus diesem Grund wird ein Schätzwert $\hat{\mathbf{x}}_{i}$ bestimmt.

Zur Rekonstruktion des Systemzustands mit Hilfe von fehlerhaften Messgrößen wird ein stationäres Kalman-Filter verwendet. Das Kalman-Filter ist ein Optimalfilter und dient der Schätzung nicht messbarer Zustandsvariablen [3, 4]. Es ist grundsätzlich ein zeitvariantes System. Bei einer zeitinvarianten Strecke konvergieren die sogenannte Kalman-Verstärkung $\mathbf{K}$ und die Fehlerkovarianzmatrizen auf stationäre Werte. Diese werden beim stationären KalmanFilter verwendet. Dadurch können zusätzlicher Berechnungs- und Speicherbedarf eingespart werden. Die Berechnungsvorschrift für den zeitinvarianten (!) Zustandsschätzer lautet

$$
\begin{aligned}
\hat{\mathbf{x}}_{i+1} & =\boldsymbol{\Phi} \hat{\mathbf{x}}_{i}+\mathbf{h} u_{i}+\mathbf{K}\left(y_{i}-\mathbf{c}^{T} \hat{\mathbf{x}}_{i}\right) \\
& =\left(\boldsymbol{\Phi}-\mathbf{K} \mathbf{c}^{T}\right) \hat{\mathbf{x}}_{i}+\mathbf{h} u_{i}+\mathbf{K} y_{i} .
\end{aligned}
$$

Die Berechnung der Kalman-Verstärkung $\mathbf{K}$ erfolgt durch

$$
\mathbf{K}=\mathbf{P}^{\star} \mathbf{c}\left(\mathbf{c}^{T} \mathbf{P}^{\star} \mathbf{c}+R\right)^{-1} \text {. }
$$

Die symmetrische Matrix $\mathbf{P}^{\star}$ ergibt sich als die positiv definite Lösung der algebraischen Matrix-Riccati-Gleichung

$$
\mathbf{P}^{\star}=\boldsymbol{\Phi} \mathbf{P}^{\star} \boldsymbol{\Phi}^{T}-\boldsymbol{\Phi} \mathbf{P}^{\star} \mathbf{c}\left(\mathbf{c}^{T} \mathbf{P}^{\star} \mathbf{c}+R\right)^{-1} \mathbf{c}^{T} \mathbf{P}^{\star} \boldsymbol{\Phi}^{T}+\mathbf{Q} .
$$

Damit ist die asymptotische Stabilität des obigen Filters gegeben, das heißt $\boldsymbol{\Phi}-\mathbf{K c}^{T}$ besitzt ausschließlich Eigenwerte im Einheitskreis.

Der durch obige Gleichungen berechnete Schätzwert $\hat{\mathbf{x}}_{i}$ wird nun anstelle der tatsächlichen Zustandsgröße zusammen mit dem Temperatursollwert $T_{3, \text { soll }}=y_{\text {soll }}$ zur Bestimmung eines Sollwertes für die adiabate Verbrennungstemperatur verwendet. Dieser wird mit den Gl. (6) und (7) in einen Erdgasmassenstrom umgerechnet. Der Sollwert wird der unterlagerten Massenstromregelung übergeben. Um bei Modellunsicherheiten etwaige stationäre Abweichungen zu eliminieren, wird zusätzlich ein I-Regler eingesetzt. Abbildung 16 gibt einen Überblick über das Regelkonzept. Im Vergleich zum vereinfachten Blockschaltbild in Abb. 2 sind nun Eingänge für die aus Messwerten bestimmten Größen Heißgasmassenstrom $\dot{m}_{H G}$ und adiabate Flammtemperatur $T_{A V}$, ist hinzugekommen.

In Abb. 17 ist das Ergebnis einer Simulation des Regelkreises dargestellt. Führungsverhalten und Störunterdrückung sind zufriedenstellend. Die guten Simulationsergebnisse werden durch den Versuch an der realen Anlage bestätigt, wie die Messdaten in den Abb. 18 und 19 zeigen. Das Führungsverhalten ist dem der Simulation ähnlich. Der Einfluss von Massenstromänderungen auf die Temperatur wird durch den Regler sehr gut unterdrückt, erst bei sehr kleinen Massenströmen ändert sich das Systemverhalten derart, dass es zu größeren Abweichungen kommt.

\section{Zusammenfassung}

Den Ausgangspunkt für diese Arbeit stellte ein einfaches Modell zur Beschreibung des thermischen Verhaltens einer Biomassefeue- 

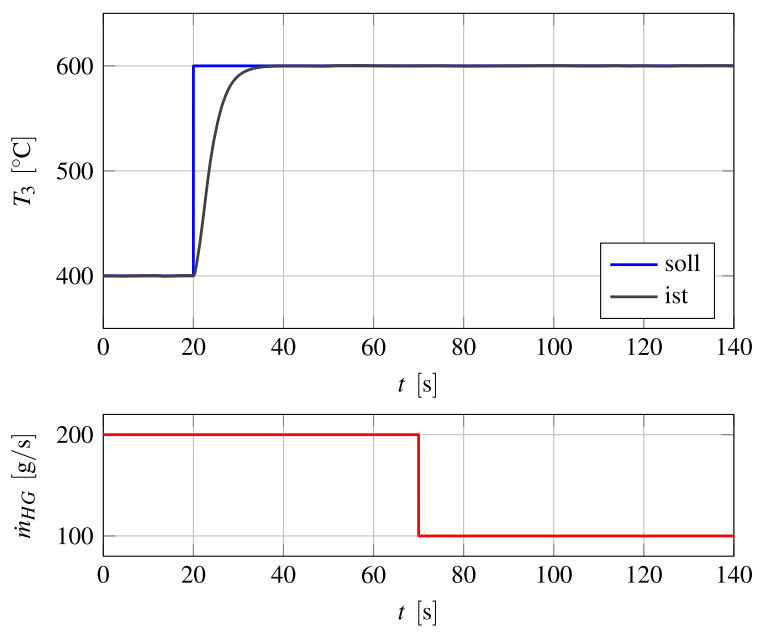

Abb. 17. Simulationsergebnisse der neuen Regelungsstrategie
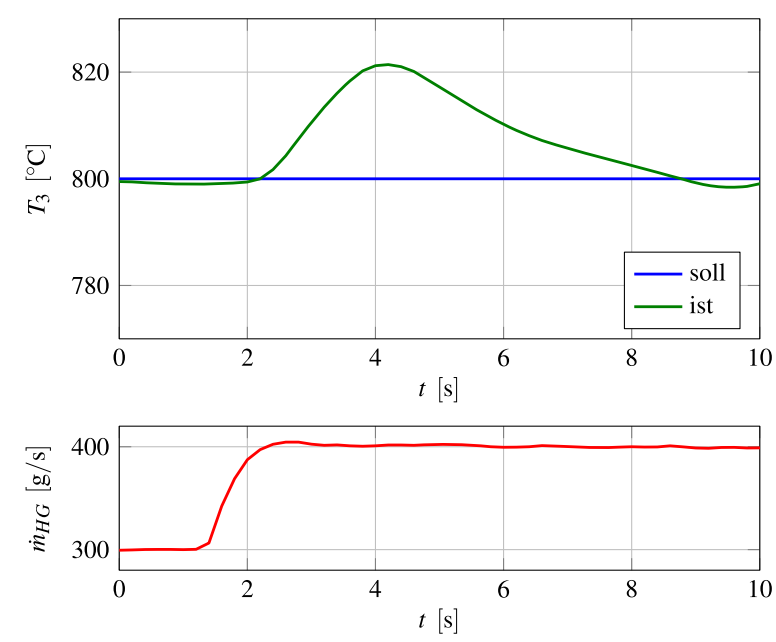

Abb. 18. Messergebnisse der neuen Regelungsstrategie (1)

rungsanlage dar. Nach einigen Modifikationen wurde ein neues Modell erstellt, das sehr gut geeignet ist, die komplexen Vorgänge in Brennkammer und Rohr eines Heißgasprüfstands abzubilden. Basierend auf diesem Modell wurde ein Regelalgorithmus entworfen, der Kenntnis über den unbekannten Systemzustand benötigt. Zur Schät-

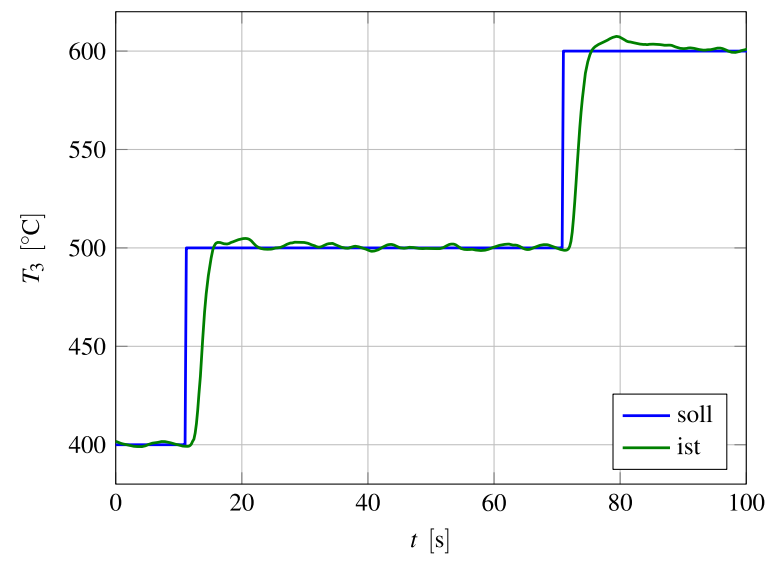

Abb. 19. Messergebnisse der neuen Regelungsstrategie (2)

zung des Zustands wurde ein stationäres Kalman-Filter eingesetzt. Durch Verwendung der neuen Regelstrategie konnte die Regelgüte gegenüber dem ursprünglich verwendeten PI-Regler deutlich verbessert werden.

\section{Danksagung}

Die Verfasser bedanken sich bei den anonymen Gutachtern für deren Verbesserungsvorschläge.

Open Access This article is distributed under the terms of the Creative Commons Attribution 4.0 International License (http://creativecommons.org/ licenses/by/4.0/), which permits unrestricted use, distribution, and reproduction in any medium, provided you give appropriate credit to the original author(s) and the source, provide a link to the Creative Commons license, and indicate if changes were made.

\section{Literatur}

1. Baehr, H. D., Kabelac, S. (2006): Thermodynamik. 13 Aufl. Berlin: Springer

2. Bauer, R. (2009): Modellierung und modellbasierte Regelungsstrategien am Beispiel einer Biomasse-Feuerungsanlage.

3. Brammer, K., Siffling, G. (1975): Kalman-Bucy-Filter: Deterministische Beobachtung und stochastische Filterung. München: Oldenbourg.

4. Gelb, A. (1974): Applied optimal estimation. Cambridge: MIT Press.

5. Gould, L. (1969): Chemical process control: theory and applications. Reading: AddisonWesley.

6. Incropera, F. P., DeWitt, D. P. (2002): Fundamentals of heat and mass transfer. 5 Aufl. New York: Wiley.

7. Profos, P., Pfeifer, T. (Hrsg.) (1997): Grundlagen der Messtechnik. 5 Aufl. München: Oldenbourg. 


\section{Autoren}
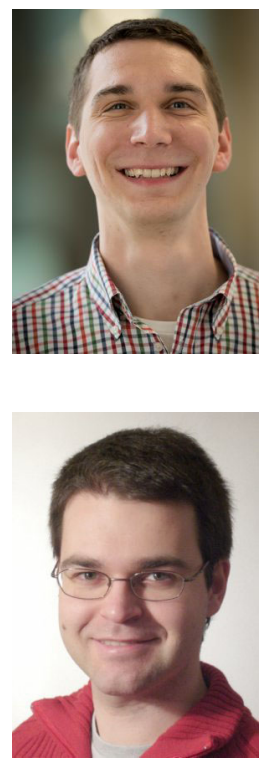

\section{Markus Freistätter}

begann nach dem Abschluss des Studiums der Telematik an der Technischen Universität Graz, Österreich, 2012 seine Tätigkeit als Projektassistent am dortigen Institut für Regelungs- und Automatisierungstechnik. In Kooperation mit der Firma Kristl, Seibt \& Co Ges.m.b.H. beschäftigt er sich mit der Modellierung und Regelung verfahrenstechnischer Prüfstände.

\section{Robert Bauer}

studierte Elektrotechnik an der Technischen Universität Graz, Österreich, und erhielt 1999 das Diplom. Er erlangte seinen Doktortitel der technischen Wissenschaften an der Fakultät für Elektrotechnik und Informationstechnik der TU Graz 2005 und erhielt 2009 die Lehrbefugnis für das wissenschaftliche Fach Prozessautomatisierung und Modellbildung. Derzeit ist er Technologiekoordinator für Modellbildung, Simulation und Regelungstechnik bei der Firma Kristl, Seibt \& Co Ges.m.b.H., seine Forschungsinteressen beinhalten die Modellierung und Regelung von nichtlinearen Mehrgrößensystemen mit besonderem Schwerpunkt auf automotive Prüfstände.

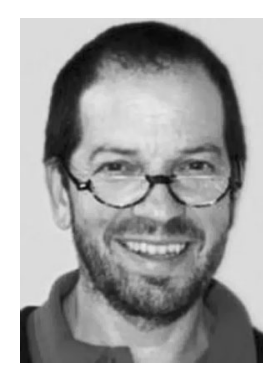

\section{Nicolaos Dourdoumas}

Nach Beendigung des Studiums der Nachrichtentechnik an der TU Berlin 1969 promovierte er 1972 an der Fakultät für Elektrotechnik der Ruhr-Universität Bochum und erlangte 1977 die venia docendi für Regelungstheorie an der Fakultät für Elektrotechnik der TU Graz. Nach der Zeit als Universitätsprofessor für Theorie der Automatisierungssysteme an der Universität Paderborn 1979-1994 war er bis zu seiner Emeritierung im Jahre 2013 Ordentlicher Universitätsprofessor am Institut für Regelungs- und Automatisierungstechnik der TU Graz. Hauptarbeitsgebiete: System- und Regelungstheorie.

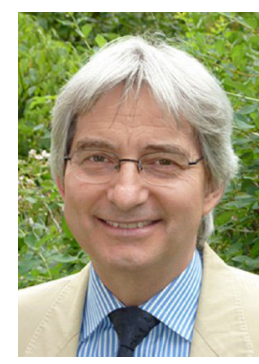

\section{Wilfried Rossegger}

studierte Elektrotechnik mit Schwerpunkt Regelungstechnik an der Technischen Universität Graz, Österreich, erhielt 1981 das Diplom und promovierte 1985 im Bereich Regelungstheorie an der TU Graz. Er ist 1981 in die als Hersteller von Prüfstandstechnik für die Automobil- und Motorenindustrie tätige Firma Kristl, Seibt \& Co Ges.m.b.H. eingetreten und seit $1995 \mathrm{ihr}$ Geschäftsführer. 